Acta Crystallographica Section D

Biological

Crystallography

ISSN 0907-4449
Hazel R. Evans, ${ }^{a}$ Daniel E.
Holloway, ${ }^{a}$ J. Mark Sutton,
Joanne Ayriss,
Shone $^{\text {b }}$ Clifford C.
and K. Ravi Acharya ${ }^{a}{ }^{\text {* }}$

a Department of Biology and Biochemistry, University of Bath, Claverton Down, Bath BA2 7AY, England, and ${ }^{\mathbf{b}}$ Health Protection Agency, Centre for Applied Microbiology and Research, Porton Down, Salisbury SP4 OJG, England
Correspondence e-mail: k.r.acharya@bath.ac.uk

(C) 2004 International Union of Crystallography

Printed in Denmark - all rights reserved

\title{
C3 exoenzyme from Clostridium botulinum: structure of a tetragonal crystal form and a reassessment of NAD-induced flexure
}

\begin{abstract}
C3 exoenzyme from Clostridium botulinum (C3bot1) ADP-ribosylates and thereby inactivates Rho A, B and C GTPases in mammalian cells. The structure of a tetragonal crystal form has been determined by molecular replacement and refined to $1.89 \AA$ resolution. It is very similar to the apo structures determined previously from two different monoclinic crystal forms. An objective reassessment of available apo and nucleotide-bound C3bot1 structures indicates that, contrary to a previous report, the protein possesses a rigid core formed largely of $\beta$-strands and that the general flexure that accompanies NAD binding is concentrated in two peripheral lobes. Tetragonal crystals disintegrate in the presence of NAD, most likely because of disruption of essential crystal contacts.
\end{abstract}

Received 15 March 2004 Accepted 12 May 2004

PDB Reference: C3 exoenzyme, tetragonal form, 1 uzi, r1uzisf.

\section{Introduction}

C3 exoenzyme from Clostridium botulinum (Aktories et al., 1987), also known as C3bot1, is the best characterized member of the $\mathrm{C} 3$ exoenzyme family, which also includes C3lim from C. limosum, C3cer from Bacillus cereus and the C3stau/EDIN subfamily from Staphylococcus aureus (reviewed by Wilde \& Aktories, 2001). These $\sim 24 \mathrm{kDa}$ enzymes specifically ADP-ribosylate and thereby inactivate mammalian Rho GTPases, a group of membrane-associated molecular switches that regulate many cell functions (Bokoch, 2000). C3bot1 specifically modifies Rho A, B and C (Braun et al., 1989; Aktories et al., 1989; Chardin et al., 1989). This process brings about a variety of cellular changes, some of which are morphological: adherent cells 'round up' (Chardin et al., 1989), monocytes spread out (Aepfelbacher et al., 1996) and neuronal PC12 cells exhibit neurite outgrowth (Rubin et al., 1988). C3bot1 also stimulates the regeneration of injured axons in vivo (Lehmann et al., 1999), highlighting its potential for the treatment of certain neural disorders (Watanabe et al., 2000). However, the role of C3 exoenzymes in the pathogenesis of bacterial disease is unclear because of the lack of an obvious cell-entry mechanism.

In common with many other bacterial toxins, C3bot1 possesses glycohydrolytic activity that splits NAD into nicotinamide and ADP-ribose, the latter of which is transferred to a specific residue of the target protein. Crystal structures of C3bot 1 in the absence and presence of NAD have indicated that the mechanism of ADPribosylation is structurally complex, involving localized conformational changes of the ADPribosylating turn-turn (ARTT) and phosphate-nicotinamide (PN) loops, plus an inde- pendent more widespread protein flexure (Han et al., 2001; Ménétrey et al., 2002). Current structural information has been obtained from two different monoclinic crystal forms, but tetragonal crystals that failed to yield useful data have also been reported (Han et al., 2001). Here, we report the production of improved tetragonal crystals, the determination of their structure and a reassessment of the conformational changes observed in the various C3bot1 structures.

\section{Experimental}

The C3bot1 gene was kindly supplied by Dr M. R. Popoff (Institut Pasteur, Paris, France). PCR was used to introduce an EcoRI site at each end of the gene and a factor Xa cleavage site immediately upstream of the first residue of the mature protein (Ala41). The forward primer was 5'-GGAATTCATCGAAGGTCGTGCTTATTCAAATACTTACCAGGAG-3' and the reverse primer was 5'-GGAATTCTTATTTAGGATTGATAGCTGTGCC-3' (EcoRI sites in bold). The PCR product was restricted with EcoRI, ligated with pBCmalE and expressed in Escherichia coli JM109 as an MBP-fusion protein as described for C3stau2 (Evans et al., 2003). The protein bound poorly to amylose affinity resin. Instead, the soluble lysate was passed through an SP-Sepharose column (Amersham Biosciences) equilibrated with $20 \mathrm{~m} M$ HEPES-NaOH pH 7.3. The protein was then eluted with an ascending $\mathrm{NaCl}$ concentration gradient and treated with factor Xa to release the C3bot1. The protein was further purified by cation-exchange and gel-filtration chromatography and then concentrated as described previously for C3stau2 (Evans et al., 2003). The final 
preparation was stored at $203 \mathrm{~K}$ in $20 \mathrm{mM}$ HEPES-NaOH pH 7.3, $20 \mathrm{~m} M \mathrm{NaCl}, 5 \mathrm{~m} M$ $\mathrm{MgCl}_{2}, 1 \mathrm{~m} M$ EDTA and was $>95 \%$ pure as judged by SDS-PAGE.

Single crystals were grown by a modification of the method of Han et al. (2001). Hanging drops containing $1.5 \mu \mathrm{l}$ C3bot1 $\left(35 \mathrm{mg} \mathrm{ml}^{-1}\right.$ ) and $1.5 \mu \mathrm{l}$ reservoir solution (18\% PEG 3350, 3\% saturated $\mathrm{Na}_{3} \mathrm{VO}_{4}$ solution, $0.15 \mathrm{M} \mathrm{NaCl}$ and $0.1 M$ imidazole/ malate $\mathrm{pH} 5.5$ ) were incubated at $292 \mathrm{~K}$ and produced tetragonal crystals within $2 \mathrm{~d}$. A range of ligand-binding experiments were conducted in which crystals were soaked for $0.5-30 \mathrm{~h}$ in reservoir solution supplemented with $9 \%$ PEG 3350 and 5-100 $\mathrm{m} M$ of either NAD, ADP-ribose or nicotinamide. Crystals tolerated nicotinamide but disintegrated within $3 \mathrm{~h}$ in the presence of either nucleotide. Intact soaked crystals were transferred briefly to a cryoprotectant composed of reservoir solution supplemented with $9 \%$ PEG 3350 and 20\% glycerol before collection of diffraction data at $100 \mathrm{~K}$ on station PX 9.6 of the SRS, Daresbury, UK.

Crystals soaked in NAD or ADP-ribose diffracted poorly. However, a high-resolution data set was collected from a crystal soaked for $30 \mathrm{~h}$ with $100 \mathrm{~m} M$ nicotinamide. The data were processed and scaled with HKL2000 (Otwinowski \& Minor, 1997) and reduced with TRUNCATE (French \& Wilson, 1978); detailed crystallographic statistics are presented in Table 1. Initial phases were determined by molecular replacement with MOLREP (Vagin \& Teplyakov, 1997) using PDB entry 1g24 molecule $A$ (Han et al., 2001) as a search model. Refinement was then carried out with REFMAC5 (Murshudov et al., 1997) using NCS restraints for the first few rounds.

\section{Figure 1}

(a) Schematic representation of C3bot1 (molecule $A$ ) from tetragonal crystals. Structural elements are coloured according to $C^{\alpha} B$ factor, ranging from 15.0 (blue) to $62.5 \AA^{2}$ (red) (mean $\left.=29.3 \AA^{2}\right)$. $(b)$ C3bot1-NAD complex. Structural elements of PDB entry 1gzf molecule $A$ are coloured as in (a), ranging from 22.7 (blue) to $77.2 \AA^{2}$ (red) (mean $=33.1 \AA^{2}$ ) and the nucleotide is shown in green in a ball-andstick representation. (c) Superposition of the $\mathrm{C}^{\alpha}$ traces from three apo C3bot1 structures. Shown in stereo are residues $A 45-A 246$ from tetragonal crystals (black), PDB entry 1g24 (red) and PDB entry 1gze (blue). (d) Superposition of the $\mathrm{C}^{\alpha}$ traces from apo-C3bot1 and the C3bot1-NAD complex. Shown in stereo are residues $A 45-A 246$ from tetragonal crystals (black) and the C3bot1-NAD complex (PDB entry 1gzf; green). (e) Superposition of the $\mathrm{C}^{\alpha}$ traces from the C3bot1-NAD and C3bot1ADP complexes. Shown in stereo are residues 45-246 from the C3bot1-NAD (PDB entry 1gzf, molecule $A$; green) and C3bot1-ADP (PDB entry 1gzf, molecule $D$; purple) complexes. Arrows denote the main-chain flexure that is necessary to convert the ADP-bound to the NAD-bound structure.
An initial round of rigid-body refinement was followed by cycles of restrained refinement, electron-density map calculation and model building using $O$ (Jones et al., 1991). Atoms for which there was negligible density were not modelled. The $F_{\mathrm{o}}-F_{\mathrm{c}}$ electron-density map showed no evidence of nicotinamide binding, but did indicate the positions of one cyclic tetrametavanadate ion, three orthovanadate ions and two glycerol molecules. Finally, water molecules were added using the $A R P / w A R P$ module

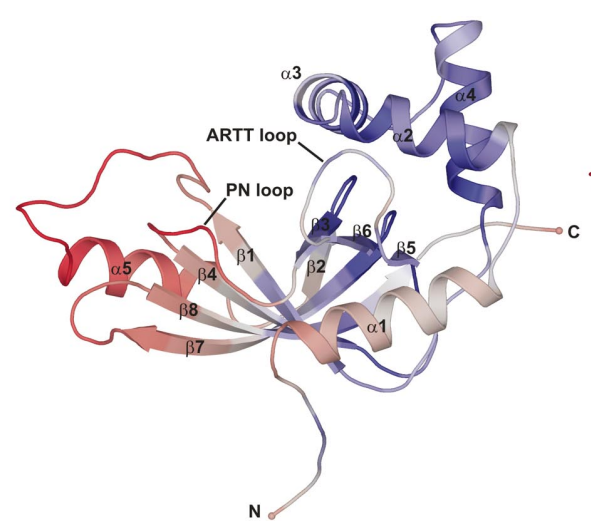

(a)

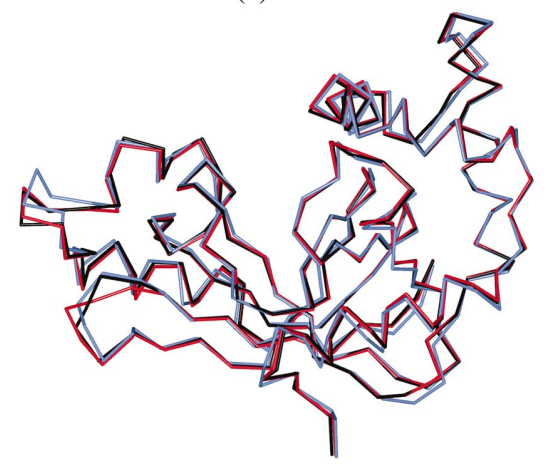

(c)

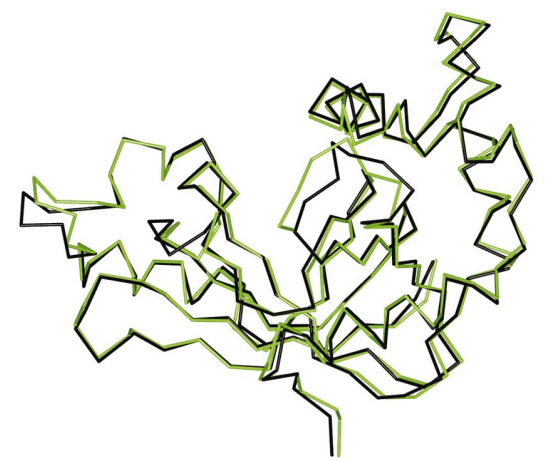

(d)

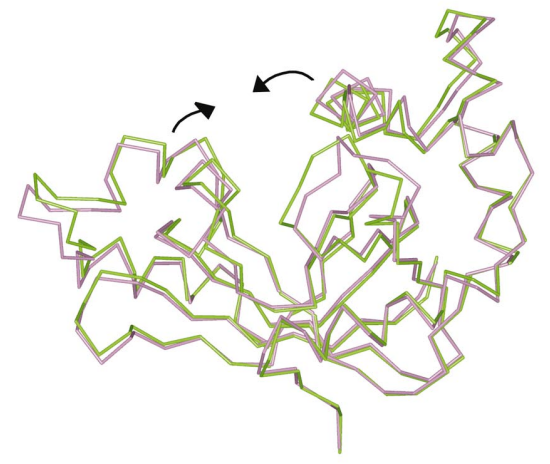

(e)

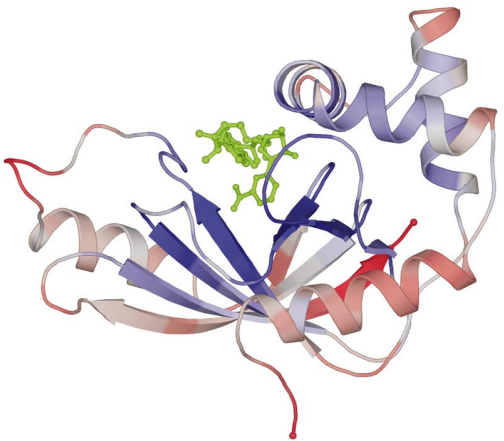

(b)
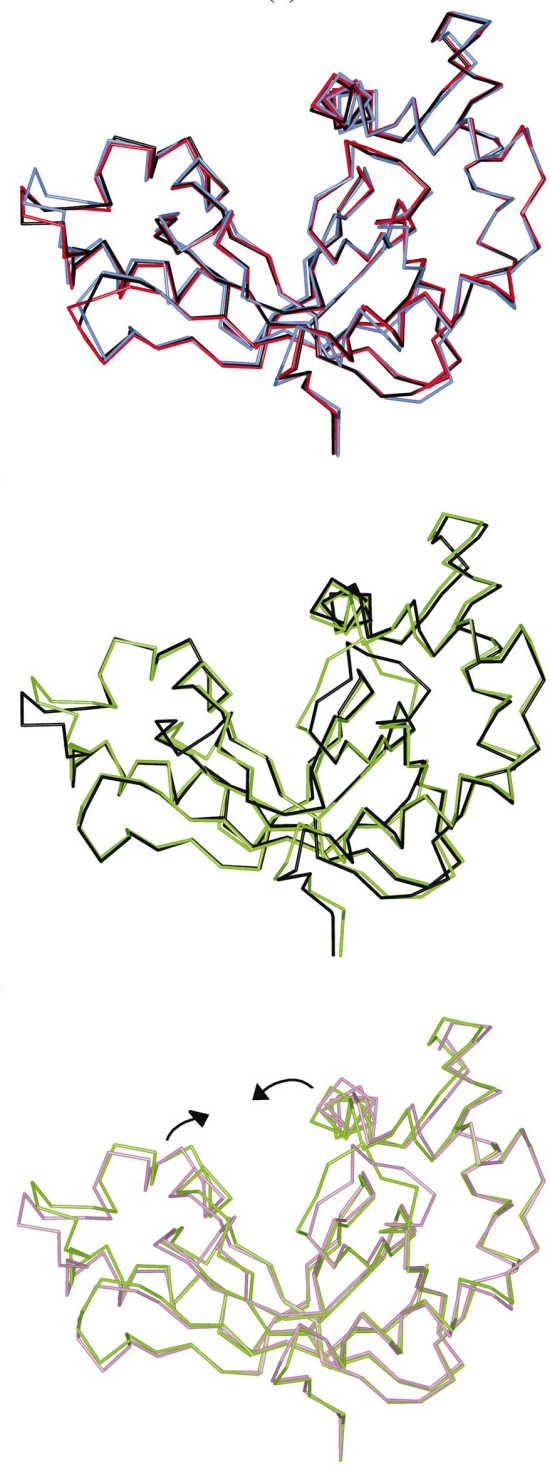
(Perrakis et al., 1999) of REFMAC5. The stereochemical quality of the structure was checked with PROCHECK (Laskowski et al., 1993). Refinement statistics are given in Table 1.

The conformational relationships between the final structure, the apo structures within PDB entries 1g24 (Han et al., 2001) and 1gze (Ménétrey et al., 2002) and the four nucleotide-bound structures comprising PDB entry 1gzf (Ménétrey et al., 2002) were examined with HINGEFIND (Wriggers \& Schulten, 1997). The program's adaptive selection algorithm was used to identify the principal rigid domain from a common set of residues (45-246). This served as the basis for all structural alignments. Figures were created with PyMOL (DeLano Scientific, San Carlos, CA, USA).

\section{Results and discussion}

\subsection{Structure determination}

The mosaicity of our best crystal $\left(\sim 0.6^{\circ}\right)$ was significantly less than that of Han et al. (2001) $\left(>1.5^{\circ}\right)$. Moreover, the diffraction data it yielded led to a clear molecularreplacement solution. A lack of $h 00$ reflections suggested the I422 space group

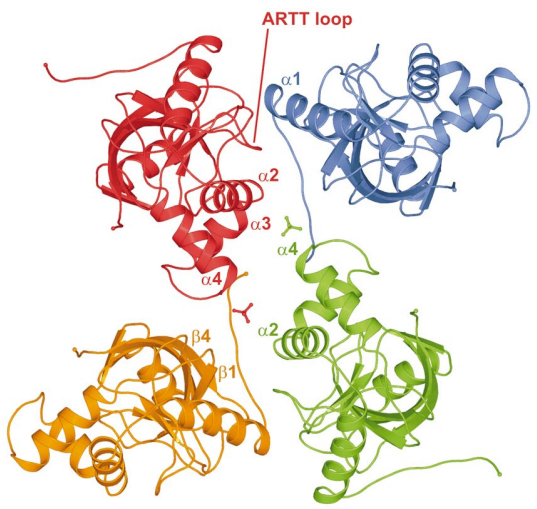

(a)
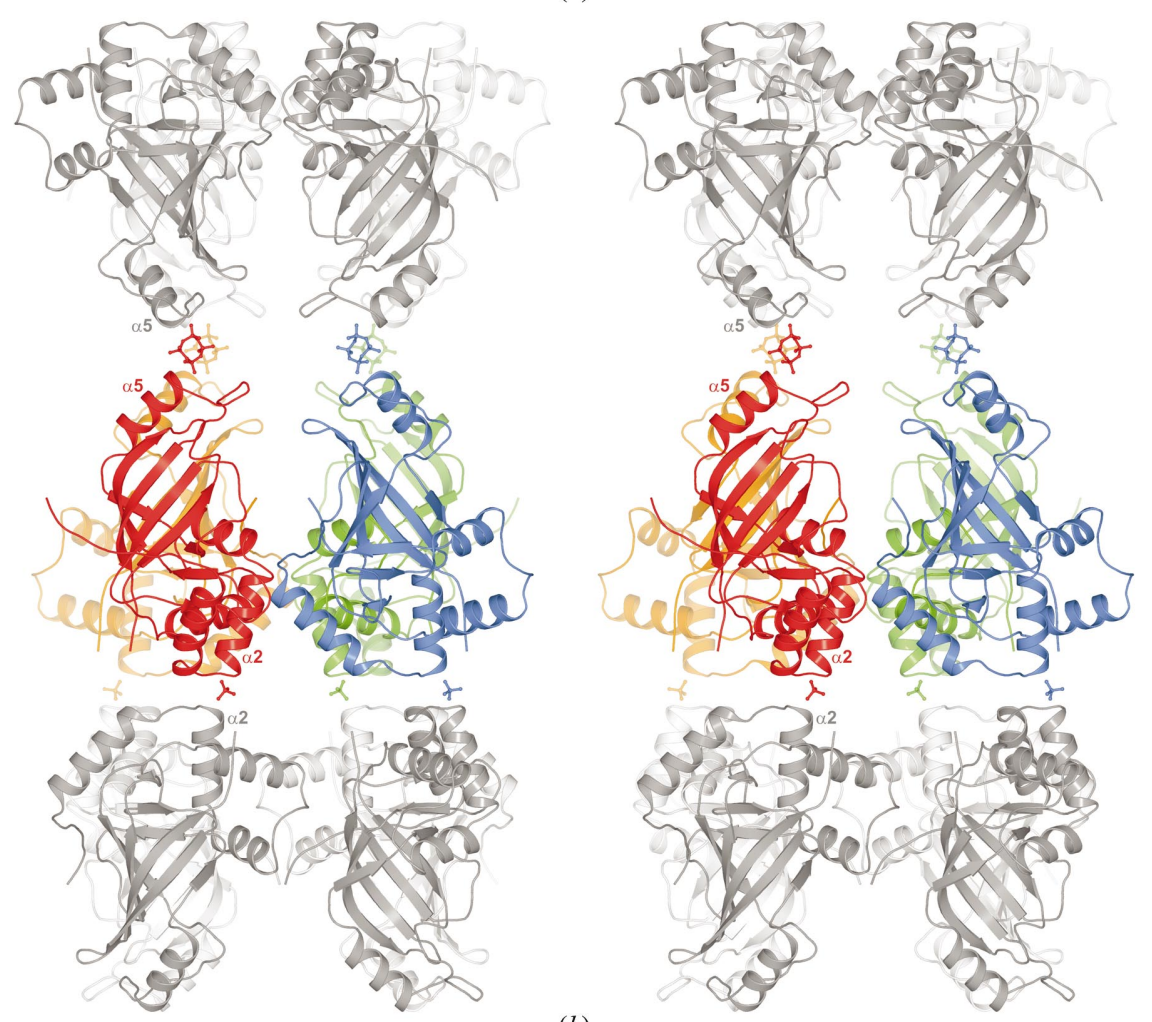

(b)

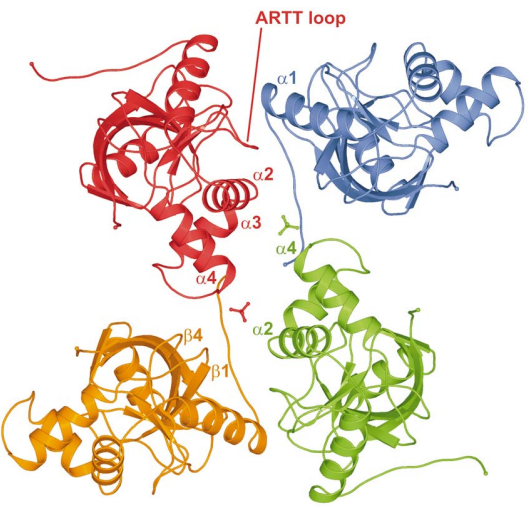

)

Figure 2

Stereoview of the packing of tetragonal C3bot1 crystals. (a) Perpendicular view of a portion of an ' $A$ ' layer. (b) In-plane view of the same layer plus adjacent ' $B$ ' layers. Symmetry-related $A$ molecules are shown in colour, $B$ molecules in grey and secondary-structure elements making crystal-packing contacts are labelled. Cyclic tetrametavanadate and orthovanadate ions are shown in ball-and-stick representation.
Table 1

Crystallographic statistics.

Values in parentheses are for the highest resolution shell $(1.96-1.89 \AA)$.

\begin{tabular}{|c|c|}
\hline \multicolumn{2}{|l|}{ Crystal system } \\
\hline Space group & I4 \\
\hline Unit-cell parameters $(\AA)$ & $\begin{array}{c}a=73.3, b=73.3 \\
c=218.2\end{array}$ \\
\hline \multicolumn{2}{|l|}{ Diffraction data } \\
\hline Resolution range $(\AA)$ & $50-1.89$ \\
\hline Reflections recorded & 683474 \\
\hline Unique reflections & 41904 \\
\hline$R_{\mathrm{sym}}^{\dagger} \dagger(\%)$ & $9.4(35.7)$ \\
\hline$I / \sigma(I)$ & $15.3(4.4)$ \\
\hline Reflections with $I>3 \sigma(I)(\%)$ & $81.8(53.8)$ \\
\hline Completeness (\%) & $91.6(91.8)$ \\
\hline \multicolumn{2}{|l|}{ Final model } \\
\hline$R_{\text {cryst }} \ddagger$ & 0.205 \\
\hline$R_{\text {free }} \S$ & 0.253 \\
\hline \multicolumn{2}{|l|}{$\begin{array}{l}\text { Dever } \\
\text { Devions from ideality (r.m.s.) }\end{array}$} \\
\hline Bond lengths $(\AA)$ & 0.019 \\
\hline Bond angles $\left({ }^{\circ}\right)$ & 1.64 \\
\hline \multicolumn{2}{|l|}{ Contents of asymmetric unit } \\
\hline Protein molecules & 2 \\
\hline Protein atoms & 3195 \\
\hline Water atoms & 308 \\
\hline Ligand atoms & 43 \\
\hline \multicolumn{2}{|l|}{ Mean $B$ factor by atom type } \\
\hline Protein & 29.9 \\
\hline Water & 33.3 \\
\hline Ligands & 52.7 \\
\hline
\end{tabular}

$\dagger R_{\text {sym }}=\sum_{b} \sum_{i}\left|I_{i}(h)-\langle I(h)\rangle\right| / \sum_{h} \sum_{i} I_{i}(h)$, where $I_{i}(h)$ and $\langle I(h)\rangle$ are the $i$ th and the mean measurements of the intensity of reflection $h$, respectively. $\$ R_{\text {cryst }}=\sum_{h}\left|F_{\mathrm{o}}-F_{\mathrm{c}}\right| / \sum_{h} F_{\mathrm{o}}$, where $F_{\mathrm{o}}$ and $F_{\mathrm{c}}$ are the observed and calculated structurefactor amplitudes of reflection $h$, respectively. $\S R_{\text {free }}$ is equal to $R_{\text {cryst }}$ for a randomly selected $5 \%$ of reflections not used in the refinement (Brünger, 1992).

described previously, but examination of crystal packing revealed that the correct space group was in fact I4. Several factors may have contributed to the improvement in diffraction quality: the different method of protein purification, the reduced precipitant concentration used for crystallization and the stabilization/dehydration of the crystals that occurred during soaking in an elevated precipitant concentration prior to data collection.

\subsection{Overall structure}

The asymmetric unit contains two C3bot1 molecules. The final model includes every residue (41-251) of each molecule, although the extremities of 23 residues from molecule $A$ and 16 from molecule $B$ could not be modelled. There are no significant differences between molecules $A$ and $B$ and their entire $C^{\alpha}$ traces show an r.m.s. deviation of just $0.13 \AA$ A. In the Ramachandran plot, $94 \%$ of the residues lie in the most favoured region, with only $0.5 \%$ (Ala184 from each molecule) in the strongly disfavoured region. This residue is also observed in an unusual conformation in several of the C3bot1 copies present in monoclinic crystals. The general topology is identical to that reported previously (Fig. 1a) and the main- 
chain trace is remarkably similar to that of PDB entry 1g24 ( $\mathrm{C}^{\alpha}$ r.m.s. deviation = $0.46 \AA$ ), the only notable differences occurring at points in the $\beta 1-\alpha 5$ and $\beta 7-\beta 8$ loops with no obvious functional significance (Fig. 1c). It is less similar to PDB entry 1gze, which shows additional localized differences distant from the active site (Fig. 1c).

\subsection{Conformational flexibility}

The distribution of main-chain $B$ factors indicates that the lobe that extends outward from the tips of strands $\beta 1, \beta 4, \beta 7$ and $\beta 8$ toward helix $\alpha 5$ is a relatively flexible part of the molecule (Fig. 1a). Within this lobe, parts of the $\beta 1-\alpha 5, \beta 3-\beta 4(\mathrm{PN})$ and $\beta 7-\beta 8$ loops have weak density and are presumed to be particularly flexible. It is of note that the conformational change that accompanies NAD binding significantly increases the relative stability of the lobe (Fig. 1b). A search for rigid domains with HINGEFIND reveals that the basis and the magnitude of the conformational change are different from those alluded to by Ménétrey et al. (2002). It shows that the molecule possesses a rigid core formed largely from the $\beta$-strands and that significant main-chain deviations are restricted to peripheral substructures, namely the $\beta 5-\beta 6$ (ARTT) loop, the PN loop and the C-terminal end of the $\beta 1-\alpha 5$ loop (Fig. $1 d$ ). The magnitude of any widespread main-chain flexure is considerably smaller than that suggested previously.

The two most extreme C3bot1 conformers observed to date are NAD-bound molecule $A$ and ADP-bound molecule $D$ from PDB entry 1gzf. Following alignment of rigid domains with HINGEFIND, a concerted movement of the $\alpha 5$ lobe is detectable (Fig. 1e), confirming the flexibility suggested by the preceding $B$-factor analysis. In addition, the parts of helices $\alpha 2, \alpha 3$ and $\alpha 4$ nearest the nucleotide also move significantly. For each half of the clasping action, the axis of movement is aligned approximately with the viewpoint of Fig. 1. However, $\varphi / \psi$ angle comparisons suggest that the flexure derives from a large number of small main-chain adjustments rather than rotation about conformational hot-spots.

\subsection{Ligand binding and crystal packing}

The binding of nicotinamide was too weak to be detected despite an experimental $\mathrm{pH}$ that was close to the optima for both the forward and reverse enzyme reactions (Habermann et al., 1991). However, soaking with $5 \mathrm{~m} M$ NAD or ADP-ribose caused crystal disintegration. This may be because several segments of the protein that move upon NAD binding (Ménétrey et al., 2002) are involved in crystal packing (Fig. 2). The packing gives the crystals a lamellar structure in which ' $A$ ' layers alternate with similarly packed ' $B$ ' layers. The majority of contacts occur within the layers, notable participants being significant portions of the ARTT loop and helices $\alpha 3$ and $\alpha 4$. The intra-layer contacts are somewhat unusual in that they involve no direct protein-protein interactions but are instead mediated by cyclic tetrametavanadate and orthovanadate ions. These involve helices $\alpha 2$ and $\alpha 5$, are distant from the nucleotide-binding site, and are likely to have less impact on nucleotide binding.

This work was supported by a postgraduate studentship held jointly between the Health Protection Agency and the University of Bath.

\section{References}

Aepfelbacher, M., Essler, M., Huber, E., Czech, A. \& Weber, P. C. (1996). J. Immunol. 157, 50705075 .
Aktories, K., Braun, U., Rösener, S., Just, I. \& Hall, A. (1989). Biochem. Biophys. Res. Commun. 158, 209-213.

Aktories, K., Weller, U. \& Chhatwal, G. S. (1987). FEBS Lett. 212, 109-113.

Bokoch, G. M. (2000). Immunol. Res. 21, 139148.

Braun, U., Habermann, B., Just, I., Aktories, K. \& Vandekerckhove, J. (1989). FEBS Lett. 243, 7076.

Brünger, A. T. (1992). Nature (London), 355, 472474.

Chardin, P., Boquet, P., Madaule, P., Popoff, M. R., Rubin, E. J. \& Gill, D. M. (1989). EMBO J. 8, 1087-1092.

Evans, H. R., Sutton, J. M., Holloway, D. E., Ayriss, J. A., Shone, C. C. \& Acharya, K. R. (2003). J. Biol. Chem. 278, 4592445930.

French, S. \& Wilson, K. (1978). Acta Cryst. A34, 517-525.

Habermann, B., Mohr, C., Just, I. \& Aktories, K. (1991). Biochem. Biophys. Acta, 1077, 253-258.

Han, S., Arvai, A. S., Clancy, S. B. \& Tainer, J. A. (2001). J. Mol. Biol. 305, 95-107.

Jones, T. A., Zou, J. Y., Cowan, S. W. \& Kjeldgaard, M. (1991). Acta Cryst. A47, 109110.

Laskowski, R. A., MacArthur, M. W., Moss, D. S. \& Thornton, J. M. (1993). J. Appl. Cryst. 26, 283-291.

Lehmann, M., Fournier, A., Selles-Navarro, I., Dergham, P., Sebok, A., Leclerc, N., Tigyi, G. \& McKerracher, L. (1999). J. Neurosci. 19, $7537-$ 7547.

Ménétrey, J., Flatau, G., Stura, E. A., Charbonnier, J.-B., Gas, F., Teulon, J.-M., Le Du, M.-H., Boquet, P. \& Ménez, A. (2002). J. Biol. Chem. 277, 30950-30957.

Murshudov, G. N., Vagin, A. A. \& Dodson, E. J. (1997). Acta Cryst. D53, 240-255.

Otwinowski, Z. \& Minor, W. (1997). Methods Enzymol. 276, 307-326.

Perrakis, A., Morris, R. M. \& Lamzin, V. S. (1999). Nature Struct. Biol. 6, 458-463.

Rubin, E. J., Gill, D. M., Boquet, P. \& Popoff, M. R. (1988). Mol. Cell. Biol. 8, 418-426.

Vagin, A. \& Teplyakov, A. (1997). J. Appl. Cryst. 30, 1022-1025.

Watanabe, Y., Morimatsu, M. \& Syuto, B. (2000). J. Vet. Med. Sci. 62, 473-478.

Wilde, C. \& Aktories, K. (2001). Toxicon, 39, 1647-1660.

Wriggers, W. \& Schulten, K. (1997). Proteins, 29, $1-14$. 